\title{
ZONAS OSCURAS EN EL SISTEMA DE ALARMA DE ADVERTENCIA DE TSUNAMI EN CHILE
}

\section{DARK ZONES IN ALARM SYSTEM OF TSUNAMI OF WARNING OF TSUNAMI IN CHILE}

\author{
Gabriel Alvarez $^{1} \quad$ Jorge Ramirez $^{1} \quad$ Lorena Paredes $^{2} \quad$ Miguel Canales $^{2}$ \\ Recibido 17 de marzo de 2010, aceptado 10 de noviembre de 2010 \\ Received: March 17, $2010 \quad$ Accepted: November 10, 2010
}

\begin{abstract}
RESUMEN
El territorio chileno cuenta con alrededor de $80.000 \mathrm{~km}$ de costa considerando el territorio insular, un dato relevante al momento de considerar la ocurrencia de un tsunami. Las autoridades chilenas, conscientes de este extenso territorio marítimo, han desarrollado un sistema de alerta de tsunami como una responsabilidad estatal y han depositado su control a la oficina nacional de emergencia -ministerio del interior (ONEMI) y en el servicio hidrográfico y oceanográfico de la armada de Chile (SHOA). En este artículo hemos realizado experiencias con el objetivo de activar los sistemas de advertencias generando eventos telúricos ficticios y/o eventos telúricos históricos capaces de desatar eventos de tsunami. También se ha propuesto una hipótesis de trabajo que permita, a través de los procedimientos establecidos por ley de la República de Chile, monitorear los tiempos de respuestas de los organismos estatales. Nuestro trabajo de investigación entrega resultados que nos permiten afirmar que existen zonas para eventos hipotéticos que podrían generar tsunamis a los cuales el sistema de alerta no sería eficiente en reaccionar. Para llevar a cabo esta investigación hemos utilizado un software llamado SLAT, basado en ecuaciones simplificadas de propagación de una onda de tsunami que nos permite obtener resultados rápidos y además hemos sometido a prueba el sistema con datos oficiales en los cuales se ha demostrado que el sistema de alerta no fue capaz de reaccionar al evento Atico 8,4 M. ocurrido en Perú.
\end{abstract}

Palabras clave: SHOA, ONEMI, tsunami, alerta, PTWC, sistema computacional, zonas oscuras.

\begin{abstract}
The Chilean territory has an extensive coastline-about $80.000 \mathrm{~km}$ of coast including the territory of its islands - which is an important fact to consider in the event of the occurrence of a tsunami. The Chilean authorities, fully aware of the vast maritime territory, have developed a tsunami warning system. This system constitutes a state responsibility, and its control has been entrusted to the national emergency office-Ministry of Interior-(ONEMI) and hydrographic and oceanographic service of Chilean navy (SHOA). This article deals with experiences carried out in order to activate the warning systems, generating fictional telluric events and / or historical telluric events capable of triggering tsunami occurrences. It also proposes a working hypothesis that will allow monitoring the response of the state agencies, through the procedures established by law in the Republic of Chile. Our research delivers results that allow us to affirm that there are areas for hypothetical events that could generate tsunamis in which the to carry out this research we have used a software called STLAT based on simplified equations of the propagation of a tsunami wave, which has allowed us to get quick results. We have also carried out tests with official data which have shown that the alarm system was not able to respond appropriately to the 8.4 M Atico event that occurred in Peru in 2001.
\end{abstract}

Keywords: SHOA, ONEMI, tsunami, warning, PTWC, software tool, dark zones.

\section{INTRODUCCIÓN}

La ubicación de Chile en el mundo se caracteriza por estar vecina al denominado cinturón de Fuego del Pacífico, denominación que ha recibido esta zona por tener los territorios con la mayor cantidad de volcanes potencialmente activos, según la organización mundial de operatorios volcánicos (WOVO). Se posee información de la existencia de movimientos telúricos a partir del año 1562 aproximadamente [7], y hasta nuestros días

\footnotetext{
1 Departamento de Geomensura. Universidad de Antofagasta. Centro de Ingeniería en Mitigación de Catástrofes Naturales (CIMCN). Av. Angamos 601. Antofagasta, Chile. E-mail: galvarez@uantof.cl

2 Departamento de Geomensura. Universidad de Antofagasta. Av. Angamos 601. Antofagasta, Chile. E-mail: jramirez@uantof.cl
} 
continuamos obteniendo información acerca de este fenómeno de la naturaleza y sus efectos secundarios, que en algunas oportunidades tienen origen marino y pueden traer como consecuencia la formación de tsunamis. La palabra tsunami es un vocablo de uso internacional, pero de origen japonés. Está compuesta de dos términos, los cuales son: TSU que significa puerto y NAMI que significa ola. El vocablo correspondiente en español es maremoto, aunque mal definido, puesto que maremoto corresponde a un terremoto cuyo epicentro está bajo el mar, el cual sacude $\mathrm{y}$ aturde los peces, pero no necesariamente genera un tren de ondas catastróficas denominadas tsunami [5].

Tabla 1. Listado de la historia de sismos de Chile.

\begin{tabular}{|c|c|c|}
\hline Año & $\begin{array}{l}\text { Magnitud } \\
\text { Richter }\end{array}$ & $\begin{array}{c}\text { Oscilaciones } \\
\text { máximas del mar }\end{array}$ \\
\hline 28 de octubre de 1562 & 8.0 & \\
\hline 8 de febrero de 1570 & $8-8.5$ & 4 m Concepción \\
\hline 16 de diciembre de 1575 & 8.5 & $4 \mathrm{~m}$ Corral \\
\hline 24 de noviembre de 1604 & 8.7 & $16 \mathrm{~m}$ Arica \\
\hline 13 de mayo de 1647 & 8.5 & \\
\hline 15 de marzo de 1657 & 8.0 & 4 m Concepción \\
\hline 8 de julio de 1730 & 8.7 & 16 m Concepción \\
\hline 25 de mayo de 1751 & 8.5 & 3.5 m Concepción \\
\hline 11 de abril de 1819 & 8.5 & $4 \mathrm{~m}$ Caldera \\
\hline 19 de noviembre de 1822 & 8.3 & $3.5 \mathrm{~m}$ Valparaíso \\
\hline 20 de febrero de 1835 & $8-8.2$ & 13 m I. Quiriquina \\
\hline 7 de noviembre de 1837 & $>8.0$ & $2 \mathrm{~m}$ Ancud \\
\hline 17 de noviembre de 1849 & 7.5 & $5 \mathrm{~m}$ Coquimbo \\
\hline 26 de mayo de 1851 & $7-7.5$ & $3 \mathrm{~m}$ Huasco \\
\hline 5 de octubre de 1859 & $7.5-7.7$ & $6 \mathrm{~m}$ Caldera \\
\hline 13 de agosto de 1868 & 8.8 & $20 \mathrm{~m}$ Arica \\
\hline 9 de mayo de 1877 & 8.8 & $21 \mathrm{~m}$ Mejillones \\
\hline 16 de agosto de 1906 & 8.3 & $1.5 \mathrm{~m}$ Valparaíso \\
\hline 4 de diciembre de 1918 & 7.6 & $5 \mathrm{~m}$ Caldera \\
\hline 10 de noviembre de 1922 & 8.4 & 9 m Chañaral \\
\hline 1 de diciembre de 1928 & 7.9 & 1.5 m Constitución \\
\hline 6 de abril de 1943 & 8.1 & $1.0 \mathrm{~m}$ Los Vilos \\
\hline 22 de mayo de 1960 & 9.5 & $15 \mathrm{~m}$ Ancud \\
\hline 28 de diciembre de 1966 & 7.8 & $0.8 \mathrm{~m}$ Caldera \\
\hline 3 de marzo de 1985 & 8.0 & $1.2 \mathrm{~m}$ Valparaíso \\
\hline 30 de julio de 1995 & 8.0 & $2.8 \mathrm{~m}$ Antofagasta \\
\hline 27 de febrero 2010 & 8.8 & 12 m Constitución \\
\hline
\end{tabular}

Fuente: SHOA. URL: http://www.shoa.cl/servicios/ tsunami/ data/tsunamis_historico.pdf

En la Tabla 1 se puede apreciar un resumen de los eventos de gran magnitud registrados en la historia reciente de Chile.

En Chile se tienen registros de terremotos desde el siglo XVI; no obstante, se ha detectado mediante estudios de columnas de sedimentos en la bahía de Mejillones, norte de Chile [10], un sismo ocurrido en 1444 probablemente, semejante en tamaño, extensión y ubicación como el ocurrido en 1877 en el norte de Chile, conocido este último por ser causante de un devastador tsunami de efectos panoceánicos. Sin embargo, los primeros datos acerca de un movimiento telúrico de gran magnitud se registran a partir del siglo XVI en Concepción en febrero de 1570, sumando a todo lo anterior un tsunami, el cual terminó por destruir aquello que quedaba en pie. En el año 1575, durante los meses de marzo y diciembre, nuevamente el país es azotado por las fuerzas de la naturaleza, según crónicas escritas de la época realizadas por padres jesuitas y políticos de la época, así como también relatos del pueblo mapuche.

Continúa a través de los siglos, la historia sísmica de Chile, siendo los más agitados el siglo XIX y el XX, con un mayor número de terremotos en este último. Se destacan los terremotos de agosto de 1906 en Valparaíso, en enero de 1939 en Chillán, este último por causar enormes daños y destrucción en infraestructura, por las numerosas muertes causadas y el corte de las comunicaciones y posterior aislamiento de las zonas afectadas [5].

No obstante, uno de los eventos más recordados en Chile y en la Cuenca del Pacífico, en cuanto a tragedias naturales se refiere, es el terremoto más grande de la historia de la humanidad en mayo de 1960, de magnitud 9,5 Mw [8]. Este terremoto causó estragos desde la ciudad de Concepción hasta la ciudad de Aysén.

La destrucción se hizo más evidente con el tsunami que se produjo en los minutos posteriores. La geografía cambió notoriamente: parte del territorio se hundió, murieron 2.000 personas, la infraestructura se derribó completamente, las comunicaciones quedaron interrumpidas y como consecuencia del sismo se originaron tsunamis en las costas de Japón, Hawai, Filipinas y Estados Unidos, entre otros [5].

Los antecedentes anteriores hacen evidente por qué Chile cuenta actualmente con servicios del sistema internacional de alerta de tsunami. En 1965, la comisión oceanográfica intergubernamental (COI) de la UNESCO estableció un sistema de alerta de ocurrencia de tsunamis (TWS) en el océano Pacífico, lugar donde se produce el sesenta por ciento de los tsunamis. Este sistema durante más de cuarenta años ha prestado servicio, asegurando a los habitantes de zonas costeras e islas previsiones tempranas y alertas contra tsunamis inminentes mejorando su eficacia con la transmisión satelital e Internet [11]. 
El presente estudio está basado en las ecuaciones simplificadas de propagación del primer tren de ondas de tsunami. La mayoría de las naciones en situación de riesgo, especialmente las islas, cuentan con sistemas locales de alerta basados en este parámetro. Es el caso de Taiwán, que preocupado por obtener un sistema de alerta que permitiera reaccionar a eventos cercanos y medianos creó un sistema de alerta local [4]. Este sistema está basado en una ubicación rápida del epicentro y posterior simulación numérica para evaluar el riesgo de tsunami. En Chile, Sudamérica, específicamente en el centro de ingeniería en mitigación de catástrofes naturales (CIMCN) de la Facultad de Ingeniería de la Universidad de Antofagasta, se ha desarrollado el software llamado SLAT [6], el cual utilizando las ecuaciones de propagación simplificadas es capaz de calcular la hora de llegada del primer tren de ondas a los lugares costeros que se estime conveniente en la cuenca del Pacífico; conocidas previamente las coordenadas del epicentro de la dislocación, la magnitud y hora de ocurrencia del terremoto, entrega entre otras informaciones de utilidad este valor aproximado que permite interpolar entre los valores entregados por pacific tsunami warning center (PTWC). Se cuenta además para este análisis con el reporte entregado por PTWC del tsunami del 23 junio del 2001 distribuido vía Internet con la información respectiva. Para tal efecto se ha calculado a través de SLAT@ [6] la hora de llegada del primer tren de ondas a los puntos de interés y analizada también la respuesta de los sistemas de alerta de tsunami para este evento.

\section{ANÁLISIS TEÓRICO}

\section{Ecuaciones simplificadas}

El desarrollo de propagación de la onda de tsunami se puede expresar en términos de balance de fuerzas [6] como se observa en la ecuación (1)

$$
r^{-1} \nabla P_{e}=a_{x}
$$

Donde $\rho$ es la densidad del agua, $P_{\mathrm{e}}$ es la presión del fondo de la columna de agua y $\alpha_{\mathrm{x}}$ es la aceleración horizontal. Esta ecuación es una simplificación de la ecuación de cantidad de movimiento en dos dimensiones horizontales (no contempla la gravitación) para flujo sin viscosidad.

La teoría de la onda solitaria, descrita en la bibliografía, indica que su altura se encontrará en cada instante sobre el nivel medio del mar en la zona estudiada. La ecuación (2) muestra la altura en función de la posición

$$
y=H *\left(\operatorname{sech}\left(\sqrt{\frac{3 * H}{4 * h^{3}} *(x-c * t)}\right)\right)^{2}
$$

Donde la velocidad se obtiene de la ecuación (3), siendo $\mathrm{x}$ la posición horizontal, $\mathrm{t}$ el tiempo, $\mathrm{H}$ la altura de la ola y h profundidad del océano:

$$
c=\sqrt{g * H *\left(1+\frac{H}{h}\right)}
$$

Estas ecuaciones permiten calcular el tiempo de demora de arribo del primer tren de ondas de origen tsunamigénicos.

En nuestro trabajo hemos utilizado el software SLAT $\odot$ [5], el cual ha simplificado estas ecuaciones. Este software nos ha permitido interpolar la hora de llegada del primer tren de ondas con mayor precisión.

Una forma de analizar una onda es determinar la razón altura onda/profundidad del agua (en adelante $\mathrm{H} / \mathrm{d}$ ); cuando esta relación es pequeña se puede explicar de la siguiente manera:

$$
\begin{gathered}
C^{2}=\frac{g * L}{2 \pi} \tanh \frac{2 \pi * d}{L} \\
\mu=2 \pi \frac{a}{T} \frac{(\cosh [2 \pi *(z+d) / L])}{\operatorname{senh} * 2 \pi *(d / L)} \cos \left(2 \pi\left(\frac{x}{L}-\frac{t}{T}\right)\right) \\
\varepsilon=-a \frac{\cosh *[2 \pi *(z+d) / L]}{\operatorname{senh}[2 \pi(d / L)]} \operatorname{sen}\left(2 * \pi\left(\frac{x}{L}-\frac{t}{T}\right)\right)
\end{gathered}
$$

En que:

$\mathrm{C}=$ velocidad de la onda.

$\mathrm{L}=$ longitud de onda.

$\mathrm{d}=$ profundidad de agua no perturbada .

$\mathrm{u}=$ velocidad horizontal de una partícula de agua en la dirección del movimiento de la onda.

$\mathrm{a}=$ amplitud de la onda sobre el nivel del agua no perturbada.

$\mathrm{T}=$ período de la onda.

$\mathrm{z}=$ distancia vertical de una partícula de agua desde la superficie del agua no perturbada.

$\mathrm{x}=$ distancia medida en la dirección del movimiento de la onda.

$\mathrm{t}=$ tiempo.

$\varepsilon=$ el desplazamiento horizontal de una partícula de agua desde su posición no perturbada. 
Los tsunamis son considerados ondas de playa de aguas someras, por estar permanentemente interferidas por el fondo marino, debido a que un medio de la longitud de onda es siempre mayor que la profundidad donde atraviesan. Por tal razón, se pueden simplificar estas ecuaciones y tratarlas como de baja amplitud para aguas someras [1].

Si d/L $\rightarrow 0$ entonces:

$$
\tanh \left[2 \pi \frac{d}{L}\right] \Rightarrow 2 \pi \frac{d}{L}
$$

Sustituyendo esta expresión en la ecuación de la velocidad $\mathrm{C}^{2}$, queda:

$$
C^{2}=\frac{g * L}{2 * n} * \frac{2 * n * d}{L}
$$

Finalmente queda :

$$
\mathrm{C}=(\mathrm{g} \cdot \mathrm{d})^{1 / 2}
$$

Velocidad de una onda de baja amplitud para aguas someras, también conocida como ecuación de Lagrange [3].

Esta ecuación permite calcular en SLAT las velocidades de propagación de la primera onda de tsunami después de un terremoto en el Pacífico; el modelo trata todos los tsunamis como si atravesaran el Pacífico con una profundidad promedio de $4.132 \mathrm{~m}$, o bien como si se desplazaran a una velocidad promedio de $725 \mathrm{~km} /$ hora. Esta velocidad se comparó con numerosos tsunamis reales ocurridos en el Pacífico [5], datos que permitieron ajustar este valor. No obstante, por ser SLAT una herramienta de emergencia se estimó apropiado disminuir las distancias de cálculo en un $10 \%$, de modo que SLAT en la mayoría de los casos adelanta la llegada de la primera onda del tren de tsunami en varios minutos. Así se tiene la seguridad de que la advertencia sea lo más oportuna posible, dejando un razonable margen de tiempo para las maniobras de evacuación.

\section{MECANISMOS DE ALERTA PARA CHILE}

El funcionamiento del sistema nacional de alarma de maremotos (SNAM) está basado fundamentalmente en la información proporcionada por una red de estaciones sismológicas y estaciones mareográficas distribuidas a lo largo del territorio chileno y/o por aquella proveniente del centro de alarmas de tsunamis del Pacífico (PTWC).

El SNAM está integrado por:

- Un organismo central, el servicio hidrográfico y oceanográfico de la armada, responsable de recolectar y evaluar los datos sísmicos y del nivel del mar y difundir las informaciones pertinentes a las autoridades civiles y marítimas.

- Organismos externos responsables de proporcionar al SHOA la información sísmica, mareográfica y oceanográfica son: el Servicio Sismológico del Departamento de Geofísica de la Universidad de Chile, la Oficina Nacional de Emergencia del Ministerio del Interior, Gobernaciones Marítimas y Capitanías de Puerto y el Pacific Tsunami Warning Center (PTWC) de Honolulú, Hawai, USA. Ver Figura 1.

- Organismos responsables de difundir la información proveniente desde el SHOA son la oficina nacional de emergencia y la dirección general de territorio marítimo y de marina mercante nacional. Dichos organismos transmiten la información a:

- Autoridades regionales.

- Naves mercantes, artefactos navales y autoridades marítimas.

- Buques y zonas navales.

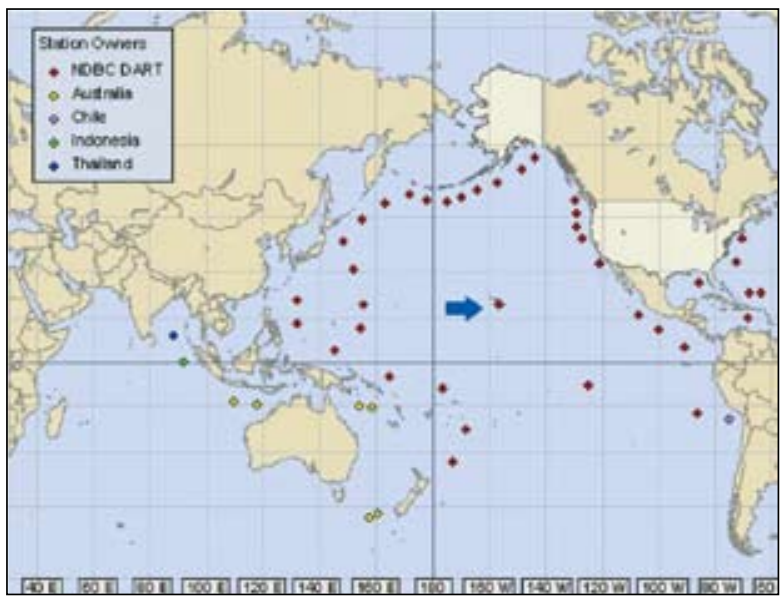

Figura 1. Estaciones de información del sistema internacional de alarma de tsunami del Pacífico.

\section{OPERACIÓN DEL SISTEMA}

El sistema entra en operaciones al ocurrir cualquier sismo que pueda generar una alerta de tsunami dentro 
del territorio nacional o al recibir un boletín informativo o de alerta de tsunami del PTWC.

En el primer caso, el SHOA procederá a recopilar con la mayor celeridad las informaciones sísmicas y del nivel del mar pertinente, con el propósito de evaluarlas para llegar a establecer la posible generación de un tsunami, lo cual, en caso afirmativo, deberá difundirse a las autoridades navales, marítimas y civiles para la adopción de las medidas preventivas que correspondan.

En el segundo caso, el SHOA, de acuerdo a las informaciones recibidas del PTWC e informaciones de las estaciones de marea insulares alejadas del continente, resolverá, previa evaluación, la difusión de las informaciones que correspondan a las autoridades nacionales ya señaladas.

La información sísmica que el SHOA evalúa proviene indistintamente de seis fuentes informativas:

a) Sistema TREMORS (Tsunami risk evaluation through seismic moment from a real-time system). Este es un nuevo sistema de detección de sismos tsunamigénicos, desarrollado por técnicos franceses del laboratorio de geofísica del comisariato de la energía atómica de Francia y adquirido por el SHOA, el cual está constituido por seis sensores sísmicos instalados en el Cerro El Roble (V Región), los cuales transmiten en tiempo real la información sísmica detectada hacia equipos instalados en el SHOA. De esta forma en el SHOA se puede analizar la información recibida por medio de software y determinar los parámetros del sismo para poder evaluar si existe riesgo de tsunami, ver Figura 2.

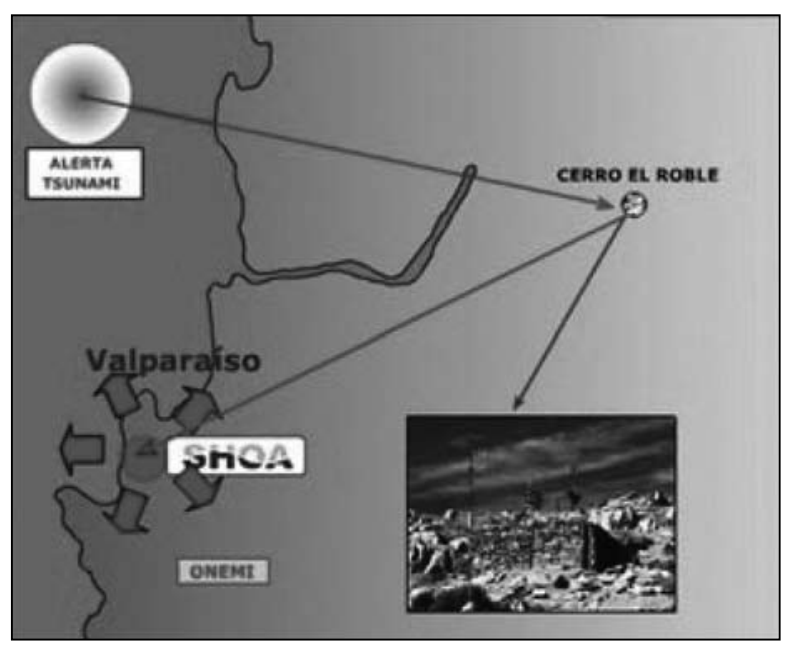

Figura 2. Ubicación del sistema TREMORS.

Fuente: Fuente SHOA. b) Sistema EMWIN. Es un sistema que permite obtener información directamente desde el NWS (national weather service) en tiempo casi real por vía satelital. Para esto el SHOA cuenta con un equipo y software, a través de los cuales se puede acceder rápidamente a la información sísmica y alertas generadas producto de un evento ocurrido en la cuenca del Pacífico.

c) Gobernaciones marítimas y capitanías de puerto. Estas al ocurrir un sismo deben emitir un mensaje destinado al SHOA de la armada, indicando su grado de intensidad, conforme con lo establecido en la directiva de la dirección general del territorio marítimo y marina mercante nacional, de fecha 28 de diciembre de 1982. Estos mensajes serán cursados por el sistema de telecomunicaciones navales con prioridad "P", salvo cuando la intensidad del sismo sea mayor que VI en la escala modificada de Mercalli, en cuyo caso los mensajes se cursarán con prioridad "O".

d) Servicio sismológico del departamento de geofísica de la Universidad de Chile. Este Servicio, en base a los registros de su red de estaciones sismológicas distribuidas a lo largo del país, informa sobre la ocurrencia de sismos sensibles en el país, determinando su ubicación, profundidad y magnitud, datos que junto a la información de intensidades reportadas son remitidos al SHOA vía telefax y también se publican en la página Web del servicio sismológico.

e) Oficina nacional de emergencia del ministerio del interior (ONEMI). Que recibe y recopila las informaciones de intensidades de los sismos producidos a lo largo del país, mediante la red de mando del ministerio del interior a través de su central de operaciones de emergencia, en enlace radial directo con las Intendencias regionales y en estrecha coordinación con los servicios públicos y privados que poseen radiocomunicaciones.

f) Pacific tsunami warning center (PTWC). Que envía boletines informativos cada vez que se produce un sismo cuya magnitud sea mayor que 6.5 y cuyo epicentro se encuentre ubicado en algún punto de la cuenca del Pacífico.

En tanto el sistema nacional de alarma de maremotos (SNAM), tras un terremoto grado 6.5 escala de Richter activa el sistema de la siguiente manera:

1) Vía satélite, el sismógrafo informa del evento a EE.UU. 
2) El procesador de la NOAA envía dos mensajes simultáneos:

2A) Alerta al computador en Chile de un posible tsunami.

2B) El procesador interroga al mareógrafo sobre cambios en el nivel del mar.

3) El mareógrafo responde.

4) El procesador califica la información y envía una señal de alarma, ver Figura 3.

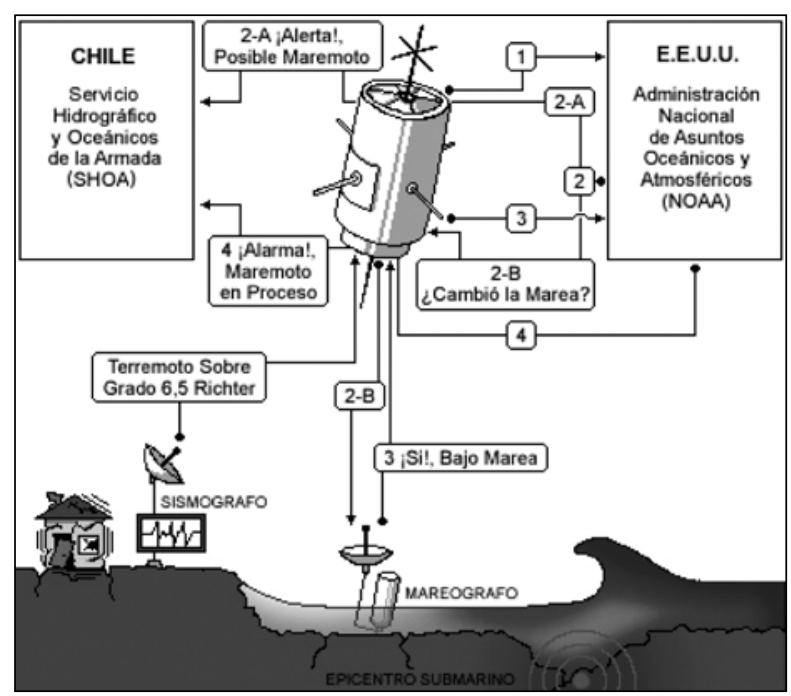

Figura 3. Esquema del sistema de alerta satelital. Fuente:http://www.puc.cl/sw_educ/geo_mar/ $\mathrm{html} / \mathrm{h} 715 . \mathrm{html}$

Criterios para la evaluación de informaciones sísmicas Las condiciones para que se genere un tsunami, producto de la ocurrencia de un sismo, son básicamente las siguientes:

a. Epicentro del sismo ubicado en el subsuelo marino o en áreas litorales del océano Pacífico.

b. Magnitud del sismo igual o superior a 7.0 en la escala de Richter.

c. Foco del sismo ubicado a menos de $60 \mathrm{~km}$ de profundidad, con respecto al fondo del mar.

Cuando se hayan reunido las tres condiciones señaladas anteriormente y además se hayan evaluado los resultados de los parámetros sísmicos entregados por el sistema TREMORS, se generarán los mensajes pertinentes por parte del SHOA, alertando a las autoridades de la posible generación de un tsunami y su hora estimada de arribo a diferentes puertos nacionales.

Si además de las condiciones indicadas se registra un tsunami en alguna estación de mareas o es informada su ocurrencia por una autoridad competente, se emitirá un nuevo mensaje confirmando la generación del tsunami con una mejor estimación de hora de arribo.

Si el tsunami no se produce y, por lo tanto, no es registrado por las estaciones de marea, se generará un mensaje cancelando su posibilidad de ocurrencia.

\section{EJERCICIO DE COMUNICACIONES}

Es importante que las comunicaciones entre los diferentes organismos que componen el sistema nacional de alerta de maremotos sean eficientes en el momento de la emergencia. Por esta razón, se envían mensajes de ejercicios a los observadores de marea en forma mensual y sin aviso previo para determinar los tiempos de transmisión y recepción de los mensajes, como asimismo mantener familiarizado al personal de comunicaciones con los procedimientos de manejo de los mensajes del sistema, y ayudar a mantener en contacto a los observadores de marea con el personal de comunicaciones de la estación de marea correspondiente. También se efectúan ejercicios de comunicaciones entre el SHOA y el PTWC y entre el SHOA y la DHN de Perú, periódicamente.

\section{Redes de comunicaciones}

Habrá dos tipos de redes de comunicaciones entre el SHOA y el resto de los organismos participantes: redes principales y auxiliares.

En un evento determinado se usarán como procedimiento normal las líneas principales, salvo que estas se encuentren inoperativas en parte o en su totalidad, en cuyo caso se recurrirá a las líneas auxiliares.

\section{Redes principales}

a) Redes del servicio de telecomunicaciones navales (telefónicas y teletipo).

b) Red de mando del ministerio del interior.

c) Teléfono comercial.

d) Telefax comercial.

Las redes del servicio de telecomunicaciones navales enlazarán al SHOA con las estaciones informantes de marea y autoridades marítimas.

La red de mando del ministerio del interior enlazará al SHOA con las oficinas regionales de emergencia.

Las redes telefónicas y telefax comerciales se consideran alternativas a las redes antes mencionadas. 


\section{Redes auxiliares}

a) Red de comunicaciones de carabineros de Chile.

b) Red de comunicaciones de la dirección general del territorio marítimo y marina mercante.

c) Red de comunicaciones de la defensa civil, bomberos, etc.

\section{Prioridad de las comunicaciones}

La prioridad de las comunicaciones debe ser tal, que permita la recepción de los mensajes e informaciones en el más breve plazo posible.

Puesto que las redes de comunicaciones que se contemplan en el sistema nacional de alarma de maremotos son redes concebidas para necesidades diferentes que las que impone este sistema, deberá actuarse con la mayor celeridad posible.

No obstante, en la red de comunicaciones de la armada se utilizarán las siguientes prioridades:

a) "P", para mensajes de información de sismos de intensidad menor que VI, emitidos por las autoridades marítimas conforme con la directiva DGTM y MM O-31/003 y durante ejercicios de maremoto.

b) "O" para mensajes de información de sismos de intensidad mayor de VI, emitidos por las autoridades marítimas, mensajes de requerimientos de información y difusión de alertas y alarmas emitidas por el SHOA, debiéndose encabezar el texto del mensaje con la palabra "URGENTE".

\section{Mensajes generados por el SHOA}

Las informaciones que el servicio hidrográfico generará, ante la ocurrencia de un sismo o informes recibidos del PTWC, estarán contenidas en cinco tipos de mensajes posibles de emitir:

1. Mensajes de requerimiento de información: Solicitan a las estaciones de marea y a la ONEMI información mareográfica y sísmica, respectivamente.

2. Mensaje de posible maremoto: Comunica la ocurrencia del sismo y se informa la hora estimada de arribo de la onda de tsunami a diferentes puertos del litoral.

3. Mensaje de confirmación de maremoto: Confirma la existencia de un tsunami. Se mejora la estimación de la hora de arribo de la onda.
4. Mensaje de cancelación: Informa que, en el caso de existir previamente un estado de posible maremoto, el sismo no tuvo una magnitud suficiente para generarlo. Y en el caso de existir una confirmación previa, este mensaje de cancelación informará que el peligro ha cesado y se puede retornar a la normalidad.

5. Mensaje de información de sismo: Comunica la ocurrencia de un sismo de magnitud igual o superior a 6.0 en la escala de Richter, indicando la ausencia de posibilidades de ocurrencia de tsunami, dada la ubicación del sismo o su baja magnitud. Tiene por objeto evitar acciones o temores innecesarios por parte de las autoridades.

\section{Mensajes generados por ONEMI}

Al ocurrir un sismo sensible en el territorio nacional, ONEMI emitirá un mensaje que contendrá la información del epicentro y magnitud del sismo proporcionada por el departamento de geofísica de la Universidad de Chile y la información de intensidades recopiladas en dicha oficina.

\section{CASO DE ESTUDIO: TERREMOTO Y TSUNAMI DE ÁTICO $M_{W}=8.4$ SUR DEL PERU}

Debido a las características de este evento telúrico los países del Pacífico fueron alertados del inicio de un tsunami. La mayoría de los daños ocurrieron en un área definida a 30 kilómetros de la línea de la costa, centrada en Ático, Perú, lugar donde el tsunami tuvo carácter destructivo.

A las 20:33:14 GMT se originó el terremoto del día 23 de junio de 2001 en Perú, según lo indicado por el instituto geofísico del Perú:

Datos del evento sísmico:

Fecha:

Sábado 23 de junio de 2001

Hora:

$$
\text { 20:33:14 GMT }
$$

Coordenadas:

$$
\begin{aligned}
& \text { Latitud } 16^{\circ} 6^{\prime} \mathrm{S} \\
& \text { Longitud } 73^{\circ} 18^{\prime} \mathrm{W}
\end{aligned}
$$

Profundidad foco:

33 kilómetros.

Magnitud:

$$
\text { 8.4 Mw. }
$$

El PTWC entregó reportes a todos los países del océano Pacífico que trabajan en conjunto, entre los cuales se encuentra Chile. En la Tabla 2 se puede ver el informe entregado. 
Tabla 2. Información efectos sismo Ático 8.4 Mw

\begin{tabular}{|c|c|c|c|c|c|}
\hline FECHA & LOCALIZ. & PARÁMETROS & $\begin{array}{c}\text { PTWC } \\
\text { ACCIÓN }\end{array}$ & $\begin{array}{c}\text { ACCIÓN } \\
\text { (TCU) }\end{array}$ & Tsu. \\
\hline \multirow[t]{2}{*}{ Junio 14} & $\begin{array}{l}\text { Andreanf } \\
\text { Island, } \\
\text { Aleutian } \\
\text { Island }\end{array}$ & \multirow{2}{*}{$\begin{array}{l}\text { LAT: } 51.2 \mathrm{~N} \\
\text { LONG: } 179.8 \mathrm{~W} \\
\text { Prof: } 18 \mathrm{~km} \\
\text { Ms: } 6.3 \\
\text { Mw: } 6.5\end{array}$} & & & \multirow[t]{2}{*}{ No } \\
\hline & $\begin{array}{r}\text { TIEMPO: } \\
\text { (TCU) } \\
19: 49 \\
\end{array}$ & & & & \\
\hline \multirow{5}{*}{ Junio 23} & Cercano & \multirow{5}{*}{$\begin{array}{l}\text { LAT: } 16.1 \mathrm{~S} \\
\text { LONG: } 73.3 \mathrm{~W} \\
\text { Prof: } 26 \mathrm{~km} \\
\text { Ms: } 8.2 \\
\text { Mw: } 8.4\end{array}$} & \multirow{2}{*}{$\begin{array}{l}\text { RWW } \\
\text { RWW } \\
\text { RWW } \\
\text { RWW } \\
\text { RWW } \\
\text { RWW } \\
\text { RWW } \\
\text { RWW } \\
\text { Canc. }\end{array}$} & \multirow{2}{*}{$\begin{array}{l}21: 14 \\
22: 12 \\
23: 10 \\
00: 11 \\
01: 14 \\
02: 13 \\
03: 10 \\
04: 14 \\
05: 03\end{array}$} & \multirow{6}{*}{ Sí } \\
\hline & $\begin{array}{l}\text { Costas } \\
\text { del Perú }\end{array}$ & & & & \\
\hline & TIEMPO: & & \multirow{5}{*}{$\begin{array}{l}\text { RWW } \\
\text { RWW } \\
\text { RWW } \\
\text { RWW } \\
\text { RWW } \\
\text { Canc. }\end{array}$} & \multirow{5}{*}{$\begin{array}{l}00: 11 \\
01: 14 \\
02: 13 \\
03: 10 \\
04: 14 \\
05: 03 \\
\end{array}$} & \\
\hline & (TCU) & & & & \\
\hline & 20:33 & & & & \\
\hline \multirow[t]{2}{*}{ Junio 24} & & & & & \\
\hline & & & & & \\
\hline \multirow{3}{*}{ Junio 26} & $\begin{array}{c}\text { Cercano } \\
\text { Costas }\end{array}$ & \multirow{3}{*}{$\begin{array}{l}\text { LAT: } 17.0 \mathrm{~S} \\
\text { LONG: } 71.1 \mathrm{~W} \\
\text { Prof: } 40 \mathrm{~km} \\
\text { Ms: } 6.6 \\
\text { Mw: } 6.8\end{array}$} & \multirow{3}{*}{ TIB } & \multirow{3}{*}{ 05:07 } & \multirow{3}{*}{ Sí } \\
\hline & del Perú & & & & \\
\hline & $\begin{array}{c}\text { TIEMPO: } \\
\text { (TCU) } \\
04: 18\end{array}$ & & & & \\
\hline \multirow[b]{2}{*}{ Julio 5} & $\begin{array}{c}\text { Sur } \\
\text { del Perú }\end{array}$ & \multirow{2}{*}{$\begin{array}{l}\text { LAT: } 15.6 \mathrm{~S} \\
\text { LONG: } 73.5 \mathrm{~W} \\
\text { Prof: } 62 \mathrm{~km} \\
\text { Ms: - } \\
\text { Mw: } 6.6\end{array}$} & \multirow[b]{2}{*}{-} & & \multirow[b]{2}{*}{ No } \\
\hline & $\begin{array}{r}\text { TIEMPO: } \\
\text { (TCU) } \\
13: 54 \\
\end{array}$ & & & & \\
\hline \multirow[b]{2}{*}{ Julio 7} & $\begin{array}{c}\text { Cercano } \\
\text { Costas } \\
\text { del Perú }\end{array}$ & \multirow{2}{*}{$\begin{array}{l}\text { LAT: } 17.6 \mathrm{~S} \\
\text { LONG: } 72.1 \mathrm{~W} \\
\text { Prof: } 16 \mathrm{~km} \\
\text { Ms: } 7.3 \\
\text { Mw: } 7.6\end{array}$} & \multirow[b]{2}{*}{ TIB } & \multirow[b]{2}{*}{$10: 14$} & \multirow[b]{2}{*}{ No } \\
\hline & $\begin{array}{c}\text { TIEMPO: } \\
\text { (TCU) } \\
9: 38\end{array}$ & & & & \\
\hline
\end{tabular}

El primer reporte fue entregado a las 21:14 GMT, 41 minutos después del terremoto. Además el informe viene complementado con una imagen que muestra los tiempos de retraso de llegada de las ondas de tsunami al todo el Pacífico, ver Figura 4.

Esta información es procesada en línea por PWTC y se va realizando su actualización en la medida que las distintas boyas distribuidas en el oceáno Pacífico informan de anormalidades en la altura del mar. Después de una serie de reportes, a las 05:03 del día 24 de junio de 2001 se canceló la alerta de tsunami. Exactamente siete horas con cuarenta y nueve minutos después que se produjo el terremoto.

Para probar la hipótesis planteada por nuestro equipo de trabajo se ha utilizado el software SLAT@ [6] de modo de interpolar el tiempo de la llegada aproximado a la ciudad de Arica $\left(18^{\circ} 28^{\prime}\right)$.
Una vez ingresados los valores, ver Figura 5, obtenemos un valor de retardo del primer tren de ondas de 33 minutos, para una distancia del epicentro de 400 kilómetros aproximadamente.

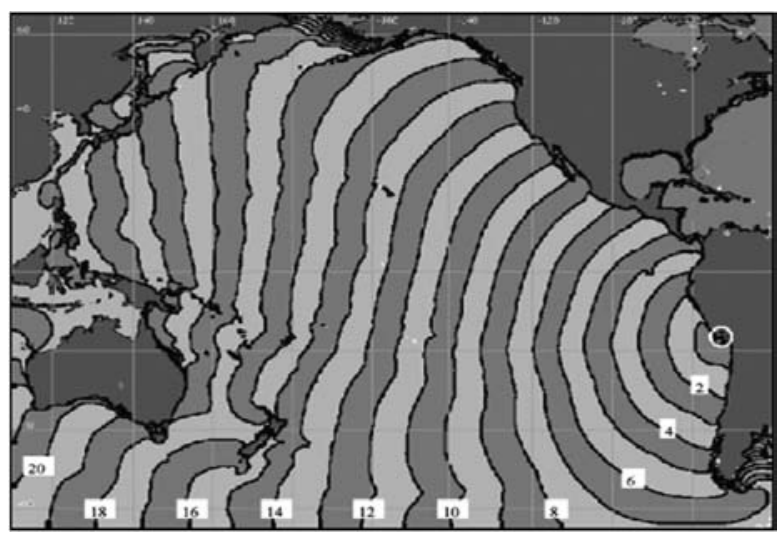

Figura 4. Tiempo de viaje de las ondas del tsunami de Perú del 23 de junio de 2001 (Fuente PWTC), las cuales están marcadas con incrementos de una hora.

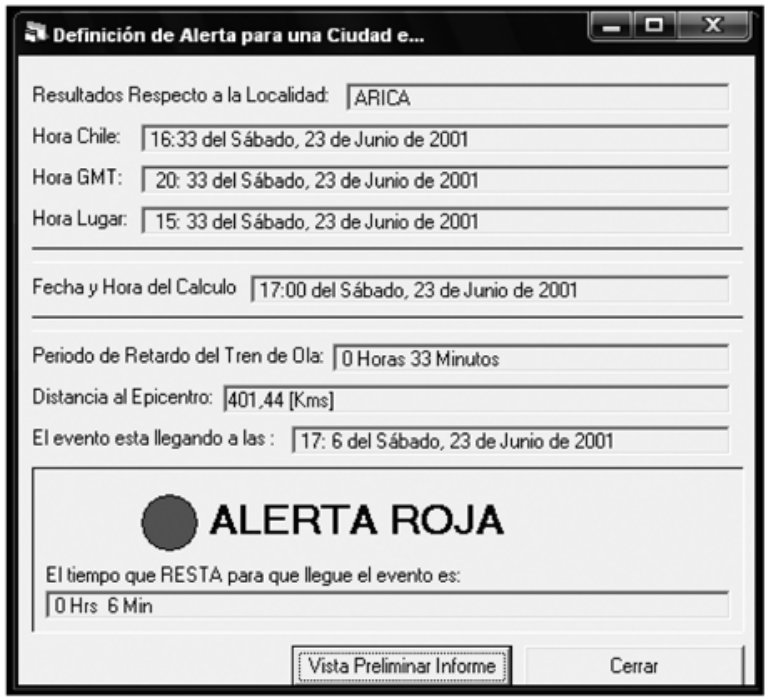

Figura 5. Cálculo hora de llegada a la ciudad de Arica del primer tren de ondas con SLAT.

A la vista de los resultados se puede apreciar que hay un lapso de retraso de la primera alerta entregada por PTWC a la ciudad de Arica de 8 minutos. Una vez llegada la primera alerta recién comienza a funcionar el sistema de alerta. Utilizando el SLATC se ha calculado para diferentes ciudades de la zona norte de Chile la llegada del primer tren de ondas. En la Tabla 3 se puede apreciar el resumen. 
Tabla 3. Resumen de resultado del cálculo para diferentes localidades de Chile.

\begin{tabular}{|l|c|c|c|c|c|}
\hline Localidad & $\begin{array}{c}\text { Periodo } \\
\text { retardo }\end{array}$ & $\begin{array}{c}\text { Fecha } \\
\text { llegada }\end{array}$ & $\begin{array}{c}\text { Hora } \\
\text { llegada }\end{array}$ & $\begin{array}{c}\text { Tiempo } \\
\text { restante }\end{array}$ & $\begin{array}{c}\text { Tipo } \\
\text { alerta }\end{array}$ \\
\hline Arica & $0: 33$ & $\mathbf{2 3 - 0 6 - 2 0 0 1}$ & $17: 06$ & $0: 06$ & Roja \\
\hline Iquique & $0: 46$ & $\mathbf{2 3 - 0 6 - 2 0 0 1}$ & $17: 18$ & $0: 18$ & Roja \\
\hline Tocopilla & $1: 01$ & $\mathbf{2 3 - 0 6 - 2 0 0 1}$ & $17: 33$ & $0: 33$ & Roja \\
\hline Mejillones & $1: 08$ & $\mathbf{2 3 - 0 6 - 2 0 0 1}$ & $17: 41$ & $0: 41$ & Roja \\
\hline Antofagasta & $1: 13$ & $\mathbf{2 3 - 0 6 - 2 0 0 1}$ & $17: 45$ & $0: 45$ & Roja \\
\hline Caldera & $1: 43$ & $\mathbf{2 3 - 0 6 - 2 0 0 1}$ & $18: 15$ & $1: 15$ & Roja \\
\hline Valparaíso & $2: 36$ & $\mathbf{2 3 - 0 6 - 2 0 0 1}$ & $19: 08$ & $2: 08$ & Roja \\
\hline
\end{tabular}

Fuente: Programa SLAT.

\section{DISCUSIÓN Y CONCLUSIONES}

Los antecedentes señalan que la primera alerta del Sistema Internacional del PTWC se emitió a las 17:14 hora local de Chile, desde el inicio del terremoto demoró 41 minutos en ser emitida, como consecuencia y dadas las características de este gran sismo se generaría un tsunami local destructor. Considerando que la señal de alerta llega al minuto 41 a los organismos centrales de emergencia, esto no quiere decir que de inmediato sea entregada a todo el litoral de Chile y Perú; es seguro que se emplean varios minutos más en conocimiento y evaluación para luego diseminar este boletín al resto del territorio. Una vez recibida en las distintas intendencias, gobernaciones, capitanías de puerto, etc., también existe un tiempo invertido en analizar y tomar la decisión de alertar y evacuar; todo el proceso dentro del territorio se estima en 20 minutos más aproximadamente, en el mejor de los casos. Por tal razón, se puede incluir dentro de esta categoría de lugares denominados "zonas oscuras" las ciudades de Iquique y Tocopilla, Chile, a pesar de que para estos lugares la alerta internacional llegaría a los organismos centrales unos +4 $y+19$ antes del arribo de la primera onda, no obstante, el tiempo ocupado en evaluar y diseminar este boletín, unos 20 minutos, las deja de igual modo en situación tardía también. En consecuencia, se puede concluir lo siguiente:

1. Costas de Tacna, Perú, el primer tren de ondas del tsunami llega 11 minutos antes que la alerta internacional, sin considerar el tiempo perdido en evaluación y diseminación.

2. Arica, Chile, el primer tren de ondas del tsunami llega 8 minutos antes que la alerta internacional, sin considerar el tiempo perdido en evaluación y diseminación

3. Iquique, Chile, el primer tren de ondas del tsunami llega 4 minutos después que la alerta internacional.
Sin embargo, si se suman los 20 minutos perdidos por el proceso de entrega del boletín por el sistema nacional y local, el tren de ondas llega 16 minutos antes que la alerta local sea recibida y distribuida en esa ciudad.

4. Tocopilla, Chile, el primer tren de ondas de tsunami llega 19 minutos después que la alerta internacional. Sin embargo, si se suman los 20 minutos perdidos por el proceso de entrega del boletín nacional y local, el tren de ondas llega un minuto antes que la alerta local sea recibida y distribuida en esa ciudad.

Se puede inferir con facilidad que ciertos sectores costeros próximos a la zona epicentral donde se generan tsunamis, ponen en jaque los sistemas de alerta y alarma de tsunami, pues están ubicados respecto del epicentro lo suficientemente cerca para que llegue muy rápidamente el primer tren de ondas de tsunami y lo suficientemente lejos como para que las ondas sísmicas se atenúen y la población no perciba el terremoto como una señal natural de alerta, lugares que se ha venido en llamar "las zonas obscuras", llamada así por la tardía recepción de una alerta de tsunami. En verdad estas zonas se extienden en un radio de unos $800 \mathrm{~km}$ desde el epicentro del terremoto que genera el tsunami.

Tomando en cuenta estos antecedentes, se piensa que estas "zonas oscuras" son un hecho real, y que en general producen la mayor cantidad de víctimas, pues los tsunamis sorprenden a la población, todo lo cual supone elaborar soluciones que permitan a los habitantes de todo el litoral chileno y sur del Perú, como también en cualquier lugar de la cuenca del Pacífico, tener una oportuna señal de advertencia cuando se generen terremotos y tsunamis de este tipo.

La segunda idea, no menos importante, es la educación; se sabe que en la escala de los elementos que permiten mitigar desastres de tsunami la educación ocupa el lugar de más alta jerarquía. Por tanto, se recomienda iniciar una campaña sostenida para los habitantes de las zonas costeras que enseñe los signos premonitorios de un tsunami, esto es, conductas anómalas del mar, franco vaciamiento o llenado sostenido, como también reconocer terremotos sensibles en el área como señal de la potencialidad de un tsunami, así como sismos que no asustan, pero por las características propias de los grandes terremotos puedan ser reconocidos también. Esta campaña debiera ser pública para la población y formal para los estudiantes de básica y media, es decir, estos contenidos debieran ser incorporados en su currículum. 


\section{REFERENCIAS}

[1] F.E. Camfield. "Tsunami Engineering". Special Reports $N^{\circ}$ 6, U.S Army Engineer Waterways Experiment Station, Coastal Engineering Research Centén, 1980.

[2] P.A. Camus. "Biogeografía marina de Chile continental". Revista chilena historia natural. Vol. $74 \mathrm{~N}^{\circ}$ 3, pp. 587-617. 2001 [citado 201003-09]. ISSN: 0716-078X. Doi: 10.4067/ S0716- 078X2001000300008. URL: http:// www.scielo.cl/scielo.php?pid=S0716078X2001000300008\&script=sci_arttext

[3] F. Espinoza. "Generación, Propagación y Efectos de Tsunamis". Armada de Chile, Dirección de Instrucción. Manuscrito. Vol. 1, pp. 1-40. Enero, 1983.

[4] C. Jr-Hung, C. Po-Fei, H. Nai-Chi and C. ChienHsin. "Tsunami arrival time database and system of Taiwan". International Workshop on Emergency Response and Rescue. October 31-November 1, 2005. URL: http://ncdr.nat.gov.tw/iwerr/doc/pdf7S $1 \% 20 \mathrm{PDF} / \mathrm{sl}-3 . \mathrm{pdf}$

[5] P.A. Lockridge. "Tsunamis in Peru-Chile". World Data Center a for Solid Earth Geophysics. Report SE-39. Vol. 97, pp. 83-92. Julio, 1985.

[6] F.J. Ramirez and P.C. Pérez. "The Local Tsunami Alert System ["SLAT"]: A Computational Tool for the Integral Management of a Tsunami Emergency". Natural Hazards. Vol. 31, Issue 1, pp. 129-142. January, 2004. DOI: 10.1023/B:NHAZ.0000020258.46865.e5
[7] P.C. Rivera. "Modelling the Asian Tsunami evolution and propagation with a new generation mechanism and a Non-Linear Dispersive Wave Model". Science of Tsunami Hazards. Vol. 25, Issue 1, January, 2006.

[8] Servicio Sismológico Departamento de Geofísica. Universidad de Chile. "Sismos importantes y/o destructivos (1570-mayo 2005)”. Catálogo. URL: http://www.sismologia.cl/seismo.html

[9] V.V. Titov and F.I. González. "Implementation and Testing of the Method of Splitting Tsunami (MOST) Model”. NOAA Technical Memorándum ERL PMEL. Vol. 112, pp. 11. November, 1997.

[10] C. Marquardt, M. Forman, G. Vargas, L. Ortlieb, J.F. Ritz, H. Philip and N. Marinivic. "Volcanic ash from the Mejillones Peninsula $\left(23^{\circ} \mathrm{S}\right)$ : Implications for the Neogene outer fore-arc stratigraphy, tectonic and volcanic relationships". $6^{\text {th }}$ International Symposium on Andean Geodynamics (ISAG 2005, Barcelona), Extended Abstracs. Vol. 1, pp. 477-480. January, 2005.

[11] B. Johannessen. "Cinco años después del tsunami del océano Índico, de la estrategia a la ejecución, los avances en los sistemas mundiales de alerta temprana contra los tsunamis y otros peligros aceánicos en 2004-2009”. Organización de las Naciones Unidas para la Educación, la Ciencia y la Cultura. Comisión Oceanógrafica Intergubernamental. Informe. Vol. 1, pp. 1-26. Enero 2009. URL: http://unesdoc.unesco.org/ images/0018/001858/1 85825s.pdf 